Evaluar, 8 (2008), 32 - 45

\title{
Fontes de Eficácia e Interesses Profissionais: relações entre pais e filhos
}

\author{
Ana Paula Porto Noronha ${ }^{1 *}$ Rodolfo Augusto Matteo Ambiel* \\ * Universidade São Francisco
}

\begin{abstract}
Resumo. O presente estudo teve por objetivo investigar as relações entre interesses profissionais de pais e filhos, e entre as fontes de auto-eficácia percebidas pelos filhos, com seus interesses e de seus pais. A amostra foi constituída por 35 estudantes de ensino médio (62,9\% do sexo feminino) e um de seus genitores $(67,6 \%$ eram mães), totalizando 70 sujeitos. Os resultados sugeriram que os interesses de pais e filhos em ciências exatas tiveram uma tendência a se relacionar moderada e significativamente e que a fonte de eficácia persuasão verbal, percebida pelos filhos, mostrou-se relacionada aos interesses dos pais nessa mesma área. Esses achados, que foram discutidos à luz de outros dados científicos encontrados na literatura estrangeira, sugerem uma tendência à transmissão de pais para filhos de padrões de interesses em ciências exatas principalmente por meios verbais. Novas pesquisas são sugeridas, com amostras maiores e com a inclusão de outros construtos.
\end{abstract}

Palavras-chave: fontes de auto-eficácia; interesses profissionais; testes psicológicos.

\begin{abstract}
The present research aimed to investigate self-efficacy sources of 35 high school students $(62,9 \%$, women) and the relationships with their own professional interests and one parent's interests $(67,6 \%$, mothers). Results suggested that parents' and children's interests in exact sciences for this sample showed a moderate and significant tendency to correlate and verbal persuasion efficacy source was related do parent's interests in the same area. These findings, that were discussed based on other scientific data found in the international literature, suggest a tendency to parents-children transmission of interests patterns in exact sciences essentially by verbal means. New studies are suggested, with bigger samples and addiction of other constructs.
\end{abstract}

Keywords: self-efficacy sources; professional interests; psychological tests.

\section{Introdução}

As pesquisas na área de Orientação Profissional (OP) no cenário brasileiro têm sido conduzidas, principalmente, em termos do levantamento dos interesses profissionais dos sujeitos, visando promover reflexões a partir dos dados obtidos e, assim, permitir uma

\footnotetext{
${ }^{1}$ Por favor dirigir la correspondencia relacionada con este artículo a: Dra. Ana Paula Porto Noronha

Rua Alexandre Rodrigues Barbosa, 45, centro. Itatiba - SP, Brasil. CEP 13251-900. Telefone/fax: (11) 4534-8118.

Email: ana.noronha@saofrancisco.edu.br
} 
possibilidade de escolha adequada. A título de ilustração pode-se citar o estudo de Noronha e Ambiel (2006), que analisou a produção científica disponível em bases de dados digitais do Brasil. Os trabalhos analisados datavam desde a década de 1950 até o ano de 2005, e observou-se que mais da metade das pesquisas que utilizaram instrumentos de avaliação lançaram mão de testes de interesses profissionais, seguidos pela avaliação de personalidade e inteligência.

Entretanto, segundo Leitão e Miguel (2004), o construto interesse profissional não está claramente definido, uma vez que desde a década de 1960 não tem sido possível constatar qualquer evolução em sua conceituação. Segundo os autores, as teorias psicológicas que buscam explicar os interesses profissionais preocupam-se mais com a operacionalização dos interesses do que com a questão de seu surgimento e desenvolvimento. Em seu trabalho de 2001, os mesmos autores já haviam discutido as definições de interesse oferecidas por vários teóricos e entenderam a de Savickas (1995) como a que melhor explicita os processos subjacentes aos interesses. Nesse particular, o autor, baseado na etimologia do termo, defende que os interesses funcionam como um componente de intermediação do indivíduo com o ambiente, assumindo uma função de motivação (Savickas, 1995). Uma vez assumida essa perspectiva, o autor hipotetiza que necessidades e valores estariam na base dos interesses, que poderiam então ser compreendidos como padrões de aceitação de determinados estímulos.

Várias teorias foram desenvolvidas e testadas no intuito de operacionalizar os interesses por intermédio de itens de tal modo que seu conteúdo expresse semanticamente as diversas facetas de tal construto. Nesse sentido, a extensa produção em nível mundial a respeito de trabalhos de autores como Holland e Strong corrobora a relevância de estudos dessa natureza para o campo da Psicologia Vocacional (Sparta, 2003). Tais autores, embora se sirvam de vertentes distintas para compor seus trabalhos, executam-no estruturalmente de forma semelhante, valendo-se de inventários para o levantamento dos interesses (Leitão \& Miguel, 2001).

Outros trabalhos, entretanto, têm sido executados no sentido de compreender de forma ampla o desenvolvimento de carreira, tomando assim os interesses como parte de um processo maior no qual também se inserem outras variáveis e construtos que devem ser levados em conta. Destaque deve ser dado à Teoria Social Cognitiva de Desenvolvimento de Carreira (TSCDC), proposta por Lent, Brown e Hackett (1994). Ela foi elaborada a partir dos pressupostos da teoria Social Cognitiva de Bandura (1986) para explicar desde o processo de 
surgimento dos interesses, considerando seus antecedentes cognitivos e contextuais, até os comportamentos de busca e engajamento em determinada profissão, exercendo assim um ponto de vista dinâmico e integrado sobre as vissicitudes dos cursos de desenvolvimento profissional. Lent e Brown (2006) ressaltam que, após mais de 10 anos da formulação da TSCDC, uma grande quantidade de pesquisas foi acumulada, garantindo seu desenvolvimento a partir de bases empíricas.

Tal teoria representa um esforço no sentido de promover uma integração entre diversos construtos, com a intenção de explicar a escolha de carreira e o desempenho acadêmico e profissional (Cupani \& Pérez, 2006). O modelo que a TSCDC preconiza considera inicialmente características pessoais e influências ambientais (Olaz, 2003), sendo que, segundo Lent e Brown (2006), essas características provêem um pano de fundo que irá determinar as experiências de aprendizagem, que por sua vez se encontram na base de formação das crenças de auto-eficácia e das expectativas de resultado.

Tais crenças são consideradas os determinantes de uma fase do processo constituída pelos interesses, os objetivos e as ações para escolha, encontrando um fim na performance bem-sucedida da carreira escolhida. Vale ressaltar que no momento no qual o indivíduo deverá fazer a definição dos objetivos e da escolha em si estão presentes outras variáveis importantes, como as barreiras e apoios percebidos, considerados pela teoria como influências ambientais proximais.

Dentro desse modelo, a auto-eficácia (Bandura, 1997) tem ocupado um espaço privilegiado e pesquisas têm sido produzidas em vários contextos, em âmbito nacional e estrangeiro, como por exemplo, em relação a dificuldades de aprendizagem (Medeiros, Loureiro, Linhares \& Marturano, 2000); adesão e sucesso de tratamentos médico (Leite, Drachler, Centeno, Pinheiro \& Silveira, 2002) e odontológico (Souza, Monteiro da Silva \& Galvão, 2002); em relação à memória no envelhecimento (Yassuda, Lasca \& Neri, 2005) e ao locus de controle e depressão em mulheres com câncer de mama (Baptista, Santos \& Dias, 2006); escrita em estudantes de diferentes níveis educacionais (Pajares \& Hobbes, 2005) e aspectos ligados à escolha de carreira (Lent \& Brown, 2006; Cupani \& Pérez, 2006).

Preconizado por Bandura (1977), o construto da auto-eficácia se refere às crenças pessoais na capacidade de organizar e executar cursos de ação requeridos para a consecução de êxito em tarefas específicas. Segundo Bandura (2001) e Olaz (2003), essas crenças não se constituem como um traço global e, portanto, não é possível avaliá-las de forma geral. 
Bandura (1997) afirma que as crenças de auto-eficácia são construídas a partir de quatro fontes de informação, a saber, experiência pessoal, aprendizagem vicária, persuasão verbal e estados afetivos e fisiológicos. A primeira fonte é tida como a mais influente, uma vez que provê uma evidência autêntica de capacidade, ou seja, a crença é construída a partir dos sucessos e fracassos da própria pessoa, ou da interpretação que ela dá às suas ações. A segunda fonte, aprendizagem vicária, informa a pessoa sobre suas capacidades a partir de transmissão de conhecimentos ou da comparação de seus atos com os de outros.

Persuasão verbal, a terceira fonte, constitui-se como uma influência externa e social, na qual um agente externo significativo convence o sujeito de sua capacidade ou incapacidade (ineficácia). Por último, estados afetivos e fisiológicos são fontes ligadas a aspectos emocionais e somáticos, tais como ansiedade, stress e estados de humor, que também podem ajudar o sujeito a perceber aspectos de sua eficácia pessoal, ajudando-o a avaliar sua confiança para realizar certas ações.

Dentre os vários estudos que se destinaram a estudar o construto em questão, alguns foram escolhidos para serem apresentados de forma mais sistemática, considerando a pertinência deles para o presente estudo. Assim, as pesquisas visaram mostrar a relação das fontes de auto-eficácia com variáveis envolvidas com a escolha profissional, tais como os interesses, e serão relatados a seguir. Nunes (2007) construiu uma Escala de Auto-Eficácia para Atividades Ocupacionais (EAAOc), composta por duas seções. Na primeira, o sujeito é inquirido a indicar três atividades que acredita realizar bem, em ordem descrescente de importância, e na segunda, responde a uma escala que avalia as fontes de eficácia relacionadas às atividades anotadas. Inicialmente, tal escala era composta por 23 itens, foi aplicada em estudantes de escolas públicas e particulares do interior do estado de São Paulo e, posteriormente, submetida à análise fatorial, que sugeriu uma estrutura interna de três fatores, sendo que a versão final ficou 18 itens.

Em tal estrutura, o primeiro fator foi composto por itens que inicialmente haviam sido escritos para perfazer as fontes Experiência Pessoal e Indicadores Fisiológicos, mas que acabaram se juntando, dando origem ao fator denominado Experiência Direta. O segundo fator, assim como a proposta teórica original, foi denominado Aprendizagem Vicária e o terceiro, Persuasão Verbal.

$\mathrm{Na}$ mesma pesquisa, a autora correlacionou tais fatores com os tipos do Self-Directed Search - SDS (Holland, Frietsche \& Powell, 1994), e encontrou correlações baixas e 
significativas entre o fator Experiência Direta com os tipos Artístico e Social, e Persuasão Verbal com Investigativo, Artístico e Social. O fator Aprendizagem Vicária não se correlacionou com nenhum tipo.

Verificar as relações entre as fontes, auto-eficácia para matemática, expectativa de resultados, interesses por cursos relaciondos à matemática e a escolha por carreiras científicas foi o própósito de Lent e Lopez (1991). Os resultados mostraram que as fontes foram preditivas significativamente, além de explicar as diferenças de gênero e a auto-eficácia para matemática. Também nessa direção, Zeldin e Pajares (2000) verificaram a influência da autoeficácia na escolha por carreiras matemáticas, científica e tecnológica, por mulheres, concluindo que aprendizagem vicária e persuasão verbal foram as fontes mais importantes. Ainda, Gainor e Lent (1998) investigaram a relação entre as fontes de auto-eficácia e os interesses por matemática e encontraram correlações significativas com as quatro fontes, na mesma ordem, de 0,44, 0,26, 0,38 e 0,43. Entretanto, é importante enfatizar que os autores utilizaram medidas de interesse por matemática, especificamente.

Pajares e Hobbes (2005) buscaram examinar a influência das quatro fontes nas crenças de auto-eficácia para escrita, por meio de dados obtidos com 1256 alunos de diferentes níveis escolares dos Estados Unidos. Os autores encontraram resultados concordantes com a teoria, uma vez que a fonte experiência pessoal de sucesso foi a que mais contou na explicação da variância, para ambos os sexos e para todos os níveis escolares.

Ainda, diferentes estudos intentaram identificar o papel de aspectos familiares na escolha profissional de adolescentes. A revisão de Lankard (1995) indicou que variáveis como nível educacional e socioeconômico, além de atitudes parentais e oportunidades concretas de desenvolvimento profissional providas pela família tendem a exercer influência significativa quanto ao processo de desenvolvimento e escolha profissional dos filhos. $\mathrm{O}$ mesmo autor enfatiza que na literatura a respeito do tema revela a preocupação em descrever e estruturar a participação dos pais no processo, bem como de orientá-los no sentido de melhor desenvolver tal papel.

Hargrove, Creagh e Burgess (2002) exploraram as relações entre padrões de interação familiar, identidade vocacional e auto-eficácia para decisão de carreira. Os resultados indicaram que a influência familiar, embora pequena, é significativa em relação aos construtos relacionados. Outros estudos mostraram que filhos do sexo masculino tendem a fazer escolhas profissionais mais próximas dos pais do que as filhas em relação às escolhas 
das mães (Steele \& Barling, 1996), ainda que os interesses dos adolescentes tendem a ser mais influenciados por pares, que pela família (Young, Antal, Basset, Post \& Valach, 1999).

As discussões acerca das relações entre variáveis familiares e interesses profissionais, apesar de não serem conclusivas, mostram que elas devem ser exploradas de forma mais sistemática, visando sua clarificação. Nesse sentido, o presente trabalho teve como objetivo verificar a magnitude das correlações existentes entre interesses profissionais de pais e filhos, e destes com as fontes de eficácia percebidas.

\section{Método}

\section{1. Participantes}

Os participantes, que ao total somaram 70, foram divididos em dois grupos. O primeiro foi formado por 35 estudantes (62,9\% do sexo feminino), com média de idade de 15,8 anos $(\mathrm{DP}=1,2)$, cursando primeira $(40 \%)$, segunda $(57,1)$ e terceira $(2,9 \%)$ séries do ensino médio de três diferentes escolas particulares do interior de São Paulo. Já o outro grupo foi composto por um dos genitores de cada participante, sendo que 67,6\% eram mães. Demais dados demográficos dos genitores não foram coletados.

\section{2. Instrumentos}

Escala de Auto-eficácia para Atividades Ocupacionais (EAAOc): Visa avaliar autoeficácia e suas fontes, tais como descritas por Bandura (1997), a saber, experiência de sucesso, aprendizagem vicária, persuasão verbal e indicadores fisiológicos. O instrumento é consituído por duas seções. Na primeira, o respondente deve anotar, em ordem decrescente de importância, três atividades que acredite fazer bem, enquanto que na segunda, deve responder a uma escala de 18 itens para cada uma das três atividades listadas na primeira seção, com vistas a avaliar a intensidade de cada fonte para as atividades listadas.

Nunes (2007) aplicou a primeira versão da escala, cuja segunda seção era composta por 23 itens, em 333 alunos (45,2\% homens) das três séries do ensino médio, em escolas públicas $(66 \%)$ e particulares do interior de São Paulo. A média de idade dos participantes foi de 15,9 anos. A partir dos dados, a autora avaliou a validade fatorial da escala, que além de sugerir a exclusão de cinco itens que não tiveram carga fatorial satisfatória, apresentou uma solução de três fatores, em que os itens relativos às fontes "Experiência Pessoal" e "Indicadores Fisiológicos" juntaram-se em um único fator, chamado Experiência Direta, composto por nove itens. Os outros dois fatores foram formados por itens que versavam sobre as duas outras fontes e assim denominados, a saber, Aprendizagem Vicária (cinco 
itens) e Persuasão Verbal (quatro itens), de acordo com o previsto pela teoria.

Visando avaliar a precisão da escala, a autora realizou estudos de consistência interna, tanto para a escala total, quanto para cada fator separadamente. Quando analisada a escala completa, com os 18 itens, o alfa obtido foi de 0,85 , enquanto que o primeiro fator indicou índice de 0,88, o segundo de 0,62 e o terceiro de 0,72 .

Escala de Aconselhamento Profissional (EAP): O EAP foi desenvolvido por Noronha, Sisto e Santos, sendo publicado em 2007. Em um primeiro momento foram construídos 220 itens a partir das descrições de 65 profissões constantes em guias profissionaise sites de universidades brasileiras, disponibilizados na Internet. Em seguida, por meio de análises heurísticas, chegou-se a uam versão composta por 61 itens que, de maneira geral, atendiam a todas as áreas. O formato da escala é Likert, cujas respostas devem variar de freqüentemente (5) a nunca (1), de acordo com o interesse do avaliando em desenvolver cada atividade.

Com relação aos estudos psicométricos, participaram 762 estudantes universitários de 13 carreiras distintas, sendo a maioria do estado de São Paulo. Dentre as evidências de validade, destaca-se o estudo de validade de construto que, por meio da análise fatorial, chegou a uma solução composta por sete fatores, a saber: Ciências Exatas (14 itens), Artes e Comunicação (14 itens), Ciências Biológicas e da Saúde (9 itens), Ciências Agrárias e Ambientais (13 itens), Atividades Burocráticas (13 itens), Ciências Humanas e Sociais Aplicadas (10 itens) e Entretenimento (seis itens), cujos índices de saturação foram superiores a 0,30 e variância explicada de 57,31\%. Vale ressaltar que alguns itens carregaram em mais de um fator, o que foi respeitado pelos autores na composição das dimensões do instrumento. A validade de critério também foi considerada, uma vez que foram comparadas as médias obtidas pelos participantes em cada dimensão em relação ao curso de cada um. Quanto à precisãoestudos de consistência interna foram realizados, sendo que os valores de alfa de Cronbach ficaram entre 0,79 e 0,94 e os de Spearman-Brown e Guttman entre 0,750,91 .

\section{3. Procedimentos}

Inicialmente, o projeto foi submetido ao Comitê de Ética em Pesquisa de uma instituição de ensino superior, e foi aprovado. O processo de coleta de dados se deu em dois momentos. Numa primeira visita às escolas, a fim de explicar aos estudantes os propósitos do estudo, foram entregues conjuntos contendo os Termos de Consentimento Livre e 
Esclarecido, juntamente com uma carta explicando os objetivos da pesquisa e uma cópia do EAP, que deveria ser respondido por um dos genitores. No total, foram distribuídos 250 conjuntos. Na semana seguinte, os estudantes cujos pais haviam consentido e preenchido o EAP respondiam aos instrumentos, primeiro EAAOc e, posteriormente, EAP. Como o consentimento e o preenchimento do instrumento por parte dos pais era pré-requisito para a participação na pesquisa, apenas 35 estudantes e os respectivos genitores compuseram a amostra, sendo que a taxa de retorno foi de 14\%, considerado satisfatória para os objetivos desse estudo.

\section{Resultados}

A seguir, serão descritos os resultados. Inicialmente, são apresentadas as estatísticas descritivas de cada instrumento, para depois, serem apresentadas as correlações entre interesses de pais e filhos, e as correlações entre as fontes de eficácia percebidas pelos filhos, seus interesses e os dos pais.

A Tabela 1 mostra as estatísticas descritivas dos escores de pais e filhos na EAP. Com relação aos escores dos filhos, ao se analisar as médias ponderadas, pode-se perceber que as dimensões Ciências Humanas e Sociais Aplicadas, e Artes e Comunicação se destacaram, em oposição a Ciências Biológicas e da Saúde e Ciências exatas, que tiveram as menores médias. Já as respostas dos pais indicaram uma predominância de Ciências Humanas e Sociais Aplicadas, e com menor destaque, Ciências Agrárias e Ambientais. Ficaram com as menores médias ponderadas, Entretenimento e Ciências Exatas.

\section{Tabela 1.}

Estatísticas descritivas de pais e filhos nas dimensões da EAP

\begin{tabular}{llllllc}
\hline & & Mínimo & Máximo & Média & Desvio padrão & Média ponderada \\
\hline \multirow{2}{*}{$\mathbf{\Sigma}$} & CieEx & 14 & 69 & 34.3 & 14.3 & 49.7 \\
& ArtCom & 15 & 64 & 39.7 & 13.2 & 62 \\
& CiBioSau & 9 & 43 & 21.7 & 10.8 & 50.5 \\
CiAgrAmb & 13 & 65 & 35.5 & 13.5 & 54.6 \\
Buroc & 14 & 57 & 33.4 & 11.4 & 58.6 \\
CiHumSoc & 12 & 41 & 26.3 & 6.9 & 64.1 \\
Entret & 6 & 28 & 16.3 & 5.7 & 58.2 \\
\hline
\end{tabular}




\begin{tabular}{llllllc}
\hline & Mínimo & Máximo & Média & Desvio padrão & Média ponderada \\
\hline & CieEx & 14 & 61 & 31.8 & 12.4 & 52.1 \\
& ArtCom & 16 & 64 & 36.5 & 12.5 & 57 \\
$\widetilde{\Xi}$ & CiBioSau & 9 & 40 & 21.7 & 8.2 & 54.2 \\
& CiAgrAmb & 17 & 54 & 37.2 & 9.8 & 68.9 \\
& Buroc & 15 & 59 & 36.1 & 11.9 & 61.2 \\
CiHumSoc & 19 & 41 & 30.9 & 5.5 & 75.4 \\
Entret & 6 & 30 & 15.3 & 6.2 & 51 \\
\hline
\end{tabular}

Legenda: CieEx: Ciências Exatas; ArtCom: Artes e Comunicação; CiBioSau: Ciências Biológicas e da Saúde; CiAgrAmb: Ciências Agrárias e Ambientais; Buroc: Atividades Burocráticas; CiHumSoc: Ciências Humanas e Sociais Aplicadas; Entret: Entretenimento.

$\mathrm{Na}$ Tabela 2, estão descritas as estatísticas relativas aos fatores da EAAOc (apenas para os filhos). Pode-se observar que o Fator 1, Experiência Direta, se sobressaiu em relação aos outros quando considerada a média ponderada, seguido pelo Fator 3, Persuasão Verbal, e Experiência Vicária (Fator 2).

Tabela 2.

Estatísticas descritivas dos fatores da EAAOc

\begin{tabular}{lccrcc}
\hline & Mínimo & Máximo & Média & Desvio Padrão & Média ponderada \\
\hline Fator 1 & 23 & 44 & 38.6 & 4.6 & 87.7 \\
Fator 2 & 6 & 21 & 13.2 & 4.2 & 62.8 \\
Fator 3 & 8 & 20 & 14.3 & 3.3 & 71.5 \\
\hline
\end{tabular}

Na Tabela 3, pode-se observar as correlações entre as dimensões da EAP, segundo as respostas de pais e filhos. Pode-se perceber que as dimensões Ciências Exatas, Artes e Comunicação, e Ciências Humanas e Sociais Aplicadas se correlacionaram de forma significativa entre si. Além disso, os escores dos filhos em Ciências Biológicas e da Saúde correlacionaram-se com os escores dos genitores em Atividades Burocráticas e Entretenimento.

As relações entre as fontes de eficácia e os interesses podem ser vistas na Tabela 4. O Fator 1 (Experiência Direta) da EAAOc se correlacionou significativamente com os interesses dos filhos em Atividades Burocráticas e o Fator 2 (Aprendizagem Vicária), com Ciências Agrárias e Ambientais, e Ciências Biológicas e da Saúde. A mesma Tabela mostra 
que o único fator da EAAOc que se correlacionou com os interesses dos pais foi o terceiro, Persuasão Verbal, que se mostrou moderadamente correlacionado com a dimensão Ciências Exatas.

\section{Tabela 3.}

Correlação entre as dimensões da EAP de pais e filhos

\begin{tabular}{|c|c|c|c|c|c|c|c|}
\hline & \multicolumn{2}{|c|}{ Dimensões/pais } & \multirow[b]{2}{*}{ CiBioSau } & \multirow[b]{2}{*}{ CiAgrAmb } & \multirow[b]{2}{*}{ Buroc } & \multirow[b]{2}{*}{ CiHumSoc } & \multirow[b]{2}{*}{ Entret } \\
\hline & CieEx & ArtCom & & & & & \\
\hline \multicolumn{8}{|c|}{ Dimensões/filhos } \\
\hline CieEx & $.45^{* *}$ & -.09 & -.05 & .12 & .02 & -.15 & .05 \\
\hline ArtCom & -.13 & $.44^{*}$ & .13 & -.02 & .19 & .21 & .31 \\
\hline CiBioSau & .16 & .24 & .25 & .33 & $.35^{*}$ & .09 & $.39 *$ \\
\hline CiAgrAmb & .14 & .33 & .17 & .30 & .22 & .20 & .25 \\
\hline Buroc & .09 & -.13 & .06 & .00 & -.19 & -.14 & -.16 \\
\hline CiHumSoc & -.10 & .21 & .06 & -.08 & .06 & $.33 *$ & .05 \\
\hline Entret & -.14 & .16 & .25 & .18 & .15 & -.01 & .14 \\
\hline
\end{tabular}

\section{Tabela 4.}

Correlações entre os fatores da EAAOc com as dimensões da EAP

\begin{tabular}{|c|c|c|c|c|}
\hline \multicolumn{5}{|c|}{$E A A O C$} \\
\hline & $E A P$ & Fator 1 & Fator 2 & Fator 3 \\
\hline \multirow{7}{*}{ 总 } & CieEx & .26 & .31 & .26 \\
\hline & ArtCom & -.17 & -.09 & -.12 \\
\hline & CiBioSau & -.19 & $.33^{*}$ & .15 \\
\hline & CiAgrAmb & .04 & $.41^{* *}$ & .20 \\
\hline & Buroc & $.34^{*}$ & .22 & .24 \\
\hline & CiHumSoc & .16 & .18 & .02 \\
\hline & Entret & -.25 & .16 & -.16 \\
\hline \multirow{7}{*}{ ప̂ } & CieEx & .30 & .05 & $.49 * *$ \\
\hline & ArtCom & .03 & .13 & .20 \\
\hline & CiBioSau & -.13 & -.05 & -.05 \\
\hline & CiAgrAmb & -.06 & .17 & .17 \\
\hline & Buroc & -.29 & -.13 & .01 \\
\hline & CiHumSoc & -.17 & -.18 & -.11 \\
\hline & Entret & .00 & .08 & .19 \\
\hline
\end{tabular}




\section{Discussão}

Tendo em vista que o presente trabalho buscou refletir sobre as relações entre interesses profissionais de pais e filhos, bem como sobre as relações desse construto com as fontes de eficácia percebida pelos filhos, pode-se afirmar que os objetivos foram alcançados, e alguns achados merecem destaque. Nunes (2007) encontrou resultados que indicam haver diferença teórica entre os construtos fontes de auto-eficácia e interesses profissionais, uma vez que as correlações encontradas foram significativas e baixas entre instrumentos avaliando os dois construtos, embora alguma comunalidade entre eles tenha sido encontrada.

Nesse sentido, o presente estudo parece indicar direção semelhante, pois das correlações entre interesses e fontes, poucas foram estatisticamente significativas, e dessas, todas foram baixas ou moderadas. Além disso, indo ao encontro de outros resultados de Nunes (2007), além dos de Pajares e Hobbes (2005) e Gainor e Lent (1998), as fontes envolvendo experiências pessoais foram as mais destacadas, no que se refere às fontes percebidas pelos filhos.

No tocante às relações entre os interesses de pais e filhos, parece haver maior magnitude correlacional entre as dimensões Ciências Exatas e Artes e Comunicação. Além disso, ao se obervar a correlação entre interesses de pais em Ciências Exatas e escores do EAAOc em Persuasão Verbal (fator 3), nota-se que tal relação foi a que se mostrou com o maior coeficiente entre todas as analisadas, podendo ser considerada uma tendência, e não uma relação casual. Considerando que os interesses de pais e filhos nessa dimensão estão também significativamente correlacionados, pode-se levantar a hipótese de que a preferência por atividades relacionadas às ciências exatas seja mais suscetível à transmissão de pais para filhos, nessa amostra, por meio oral, ou ainda, que os pais que se interessem mais por tais atividades sejam mais propensos a incentivar verbalmente os filhos nesse sentido.

Tais resultados parecem encontrar respaldo nos achados de Hargrove, Creagh e Burgess (2002), que indicam que os padrões de interação familiar têm uma pequena, mas, significativa influência no estabalecimento da identidade vocacional. Ainda, os resultados de Zeldin e Pajares (2000) e Gainor e Lent (1998), mostram a importância da fonte Pesuasão Verbal em questões relacionadas aos interesses por matemática.

Em síntese, discussões sobre a formação dos interesses profissionais e seus antecedentes cognitivos e comportamentais têm tomado pouco espaço na literatura (Leitão \& 
Miguel, 2004), apesar da importância de tal informação para os processos de avaliação vocacional. Os dados encontrados nesse estudo não são de forma alguma conclusivos, uma vez que a amostra utilizada foi pequena para fins de generalização, mas indicam caminhos possíveis para novas pesquisas, visando confirmar e esclarecer as relações sugeridas. Para estudos futuros, sugere-se a inclusão de medidas de estilos parentais e auto-eficácia para decisão de carreira, a fim de se apronfudar o estudo sobre a influência familiar nos processos de decisão dos adolescentes.

\section{Referências}

Bandura, A. (1977). Self-efficacy: toward a unifying theory of behavioral change. Psychological Review, 84, pp. 191-215.

Bandura, A. (1986). Social foundations of thought and action: a social cognitive theory. Englewood Cliffs, NJ: Prentice-Hall.

Bandura, A. (1997). Self-efficacy: The exercise of control. New York: W. H. Freeman and Company.

Bandura, A.(2001) Guía para la construcción de Escalas de Autoeficacia.(versión revisada). EEUU: Universidad de Stanford.

Baptista, M. N., Santos, K. M., \& Dias, R. R. (2006). Auto-eficácia, lócus de controle e depressão em mulheres com câncer de mama. Psicologia Argumento, 24(44), 27-36.

Cupani, M., \& Pérez, E. R. (2006). Metas de elección de carrera: contribución de los interesses vocacionales, la autoeficacia y los rasgos de personalidad. Interdisciplinaria, 23(1), 81-100.

Gainor, K., \& Lent, R. (1998). Social cognitive expectations and racial identity attitudes in predicting the math choice intentions of black college students. Journal of Counseling Development, 45(4), 403-413.

Hargrove, B. K.; Creagh, M. G. \& Burgess, B. L. (2002). Family interaction patterns as predictors of vocational identity and career decision-making self-efficacy. Journal of vocational behavior, 61(2).

Holland, J.L., Frietsche, B. A., \& Powell, A.B. (1994). SDS Self-Directed Search Technical Manual. Florida: Psychological Assesment Resources, Inc.

Lankard, B. A. (1995). Family role in career development. ERIC Digest n. 164. ERIC Clearinghouse on Adult, Career, and Vocational Education. Http://ericacve.org.

Leitão, L. M., \& Miguel, J. P. (2001). Os interesses revisitados. Psychologica, 26. pp. 79-104.

Leitão, L. M., \& Miguel, J. P. (2004) Avaliação dos interesses. Em: L. M. Leitão (org.), Avaliação psicológica em orientação escolar e profissional (pp. 179-262). Coimbra:Quarteto.

Leite, J. C. C.; Drachler, M. L.; Centeno, M. O.; Pinheiro, C. A. T.; \& Silveira, V. L. (2002). Desenvolvimento de uma Escala de Auto-Eficácia para Adesão ao Tratamento Anti-Retroviral. Psicologia: Reflexão e Crítica, 15(1), pp. 121-133.

Lent. R. W., \& Lopez, F. G. (1991). Mathematics self-efficacy: Sources and relation to science-based career choice. Journal of Counseling Psychology, 38(4), 424-431. 
Lent, R. W., \& Brown, S. D. (2006). On conceptualizing and assessing social cognitive constructs in career research: a measurement guide. Journal of career assesment, 14(1), pp. 12-35.

Lent, R., Brown, S. D., \& Hackett, G. (1994). Toward a Unifying Social Cognitive Theory of Career and Academic Interest, Choice and Performance. Journal of Vocational Behavior, 45, 79-122.

Medeiros, P. C.; Loureiro, S. R.; Linhares, M. B. M.; \& Marturano, E. M. (2000). A Auto-Eficácia e os Aspectos Comportamentais de Crianças com Dificuldade de Aprendizagem. Psicologia: Reflexão e Crítica, 13(3), pp.327-33

Noronha, A. P. P., \& Ambiel, R. A. M. (2006). Orientação profissional e vocacional: análise da produção científica. PsicoUSF, 11(1), pp. 75-84.

Noronha, A. P. P., Sisto, F. F., \& Santos, A. A. A. (2007). Escala de Aconselhamento Profissional: manual técnico. São Paulo: Editora Vetor.

Nunes, M. F. O. (2007). Escala de Fontes de eficácia percebida: aplicação com jovens em momento de escolha profissional. Dissertação de mestrado não-publicada, Universidade São Francisco, Itatiba, SP.

Olaz, F. (2003). Modelo social cognitivo del desarrollo de carrera. Evaluar, 3, 15-34

Pajares, F., \& Hobbes, C. (2005). Sources of Writing Self-Efficacy Beliefs of Elementary, Middle, and High School Students. Trabalho apresentado no Encontro da American Educational Research Association, Montreal, Canadá.

Savickas, M. L. (1995). Examining the personal Meaning of inventoried interests during career counseling. Journal of Career Assesment, 3(2), 188-201.

Souza, G. A. de; Monteiro da Silva, A. M.; \& Galvão, R. (2002) A auto-eficácia como mediadora da melhora em índices clínicos de saúde oral. Pesquisa Brasileira em Odontopediatria e Clínica Integrada, 16(1), pp. 57-62

Sparta, M. (2003). O desenvolvimento da Orientação profissional no Brasil. Revista Brasileira de Orientação Profissional, 4 (1/2), 1-11.

Steele, J., \& Barling, J. (1996), Influence of maternal gender-role beliefs and role satisfaction on daughter's vocational interests. Sex Roles, 34, 637-648.

Yassuda, M. S.; Lasca, V. B.; \& Neri, A. L.(2005). Meta-memória e Auto-eficácia: Um Estudo de Validação de Instrumentos de Pesquisa sobre Memória e Envelhecimento. Psicologia: Reflexão e Crítica, 2005, 18(1), 78-90.

Young, R. A., Antal, S., \& Basset, M. E., Post, N. D., \& Valach, L. (1999). The joint actions of adolescents in peer conversations about career. Journal of adolescence, 22, 527-538.

Zeldin, A. L., \& Pajares, F. (2000). Against the odds: Self-efficacy beliefs of women in mathematical, scientific, and technological careers. American Educational Research Journal, 37(1), 215-246. 